\title{
EVALUATION OF OXIDATIVE STRESS AND ANTIOXIDANT STATUS BETWEEN TYPE II DIABETES PATIENTS AND HEALTHY POPULATIONS
}

\author{
SUDHARSHAN REDDY NELLI ${ }^{1 *}$, NILESH KUMAR SHARMA ${ }^{1}$, MANOJ KUMAR P², SURYA S SINGH ${ }^{3}$ \\ ${ }^{1}$ Department of Cancer and Translational Research, Cancer and Translational Research Lab, Dr. D. Y. Patil Biotechnology and \\ Bioinformatics Institute, Dr. D. Y. Patil Vidyapeeth, Pune - 411 033, Maharastra, India. ${ }^{2}$ Department of Biochemistry, Osmania University, \\ Hyderabad - 500 007, Telangana, India. ${ }^{3}$ Department of Biochemistry and Coordinator, DBT-OU-ISLARE, University College of Science, \\ Osmania University, Hyderabad - 500 007, Telangana, India. Email: nsreddy.tc@gmail.com
}

Received: 16 March 2017, Revised and Accepted: 25 May 2018

\section{ABSTRACT}

Introduction: The aim of the present study is to profile the red blood cells (RBC) antioxidative enzymes, catalase (CAT), glutathione peroxidase (GPx), and superoxide dismutase (SOD) level in Type II diabetes mellitus patients in comparison to healthy volunteers in the South Indian population.

Methodology: A prospective, observational, case-control study was conducted for 1 year with a total of 120 patients including 90 Type II diabetes patients (case group) and 30 healthy volunteers (control group). Blood was collected from these volunteers, and RBC levels of CAT, GPx, and SOD were estimated. In addition, they were also monitored for the fasting blood glucose, glycated hemoglobin (HbA1c), and postprandial blood glucose. Data were statistically analyzed applying unpaired t-test and Pearson correlation with the statistical significance of $\mathrm{p}<0.05$.

Results: The diabetes patient group showed significant higher levels of glycated hemoglobin, fasting blood glucose and postprandial blood glucose $(\mathrm{p}<0.0001)$. There was a significant lower level in the RBC levels of superoxide dismutase in case group compared to control group $3859.00 \pm 381.8$ (mean+SD) and 5862.7 \pm 209.45 (mean+SD) Units per gram Hb, (t-value 27.35, p-Value <0.0001). Catalase and Glutathione peroxidase RBC levels also showed significant lower levels in the case group compared to the control group (catalase $212.7 \pm 19.08$ (mean \pm SD) and 396.47 \pm 10.83 (mean \pm SD) Units per gram Hb; $T$ value $=50.07$ and $p<0.0001$ )(Glutathione peroxidase11.7 \pm 01.09 (mean $+S D$ ) and $18.6 \pm 01.00$ (mean $+S D$ ) Units per gram $\mathrm{Hb} ; \mathrm{t}$ value $=30.26$ and $p<0.0001$ ).

Conclusion: A significant reduction in RBC levels of antioxidative enzymes, CAT, GPx, and SOD was observed in the South Indian Type II diabetes patient population.

Keywords: Diabetes, Antioxidants, Superoxide dismutase, Glutathione peroxidase, Human volunteers.

(c) 2018 The Authors. Published by Innovare Academic Sciences Pvt Ltd. This is an open access article under the CC BY license (http://creativecommons. org/licenses/by/4. 0/) DOI: http://dx.doi.org/10.22159/ajpcr.2018.v11i9.25998

\section{INTRODUCTION}

Diabetes mellitus is a cluster of metabolic disorder characterized by hyperglycemia symptoms and abnormalities in protein, fat, and carbohydrate metabolism. It results from insufficiencies in insulin exudation or sensitivity or both [1]. Diabetes is becoming more prevalent and widespread illness in both developed and developing countries at a shocking rate. In the past three decades, diabetes has altered from being a minor disease affecting the elderly to one of the chief causes of morbidity and mortality in the young and middle-aged population [2]. The WHO report predicts that more than 180 million people might be affected by this disease globally and is projected to double by 2030 making Type II diabetes as a single most leading disease affecting many people [3]. Type I and Type II diabetes escalate the risk of other health disorders such as vascular complications [4]. The previous studies have indicated that there is a strong correlation between oxidative stress (OS) and diabetes complications.

OS is defined as the tissue injury resulting from an inequity between an extreme generation of oxidant composites (superoxide, hydrogen peroxide, alkoxy radical, and hypochlorous acids) and insufficient antioxidant (Vitamin E and C, N-acetylcysteine, L-arginine, Glutathione, and Glutathione S-transferase) defense mechanisms [5,17]. OS can play an important role in the etiology of diabetes and microvascular and macrovascular complications of diabetes. Uncontrolled diabetes leads to increased production of free radicals, particularly reactive oxygen species (ROS) arising from glucose auto-oxidation and protein glycosylation. These ROS impact many biomolecules such as nucleic acids, proteins, and lipids by altering their nature and function; thus, leading to cell damage in diabetes mellitus. Thus, the multimodal mechanisms leading to diabetes has strong correlation with OS [6].

OS markers: ROS compounds are extremely sensitive and have halflives of only a few seconds; hence, their in vivo measurement to quantify OS is challenging. The superoxide dismutase (SOD) enzyme catalyzes the reduction of superoxide $\left(. \mathrm{O}_{2}^{-}\right)$into $\mathrm{H}_{2} \mathrm{O}_{2}$ and $\mathrm{O}_{2}$ as the initial stage of the antioxidant pathway, thereby playing a vital role in the removal of $\mathrm{O}_{2}$. Catalase (CAT) exists principally in the peroxisomes of mammalian cells as a homotetramer each consisting of NADPH and heme group at its reactive center [16]. The important action for CAT in the ablation of atherosclerosis comes from the study that has used mice overexpressing CAT [7]. Clinical and preclinical studies are suggesting a vital role for glutathione peroxidase (GPx) in the defense against atherosclerosis associated with diabetic patients. The GSH reduces lipid peroxidases and hydrogen to water and their equivalent alcohol. Moreover, GPx also helps eliminate harmful ONOO. Thus, GPx has been implicated to protect against many disease pathologies by metabolizing three major ROS, Lipid peroxide (LOOH), H2O2, and ONOO- [8]

If ROS production overcomes the antioxidant protection of the cell either because of excessive formation of oxidants or reduction of antioxidant defenses, various cellular molecules and their functions may be compromised. The main cellular targets are proteins, lipids, and DNA. In adding, gene expression and protein function can be controlled by oxidants [9]. The aim of the present study is to profile the $\mathrm{RBC}$ antioxidative enzymes, SOD, CAT, and GPx level in Type II diabetes patients in comparison to healthy volunteers in the South Indian population. 
Experimental protocols

\section{Materials}

Instruments and chemicals used: Spectrophotometer (BT-9300 ${ }^{\text {st }}$ Laser Auto Analyzer), colorimeter, centrifuge, water bath thermostatic, $\mathrm{pH}$ meter, cyclomixer, micropipettes $(100 \mu \mathrm{L}, 1000 \mu \mathrm{L})$, Eppendorf tubes, test tubes, beakers and chemicals, disodium hydrogen phosphate, DTNB (5,5'-dithiobis (2-nitrobenzoic acid), reduced glutathione, methanol, sodium dihydrogen phosphate, thiobarbituric acid, trichloroacetic acid, disodium EDTA, and CDNB(1-chloro-2,4-dinitrobenzene). All the chemicals and reagents procured from Sigma-Aldrich otherwise stated.

\section{Methodology}

This is a prospective, observational, case-control study conducted around 1 year in outpatient wards of tertiary care teaching hospital. Before initiating the study, Institutional Human Ethical Committee (IHEC no: DRSER 0733/02) permission was obtained. The patients were admitted to outpatient department review on the daily basis and were scrutinized for inclusion criteria such as patient's diagnosis with Type II diabetes mellitus tests and healthy volunteers. The patients with liver failure, severe comorbidity disease (other disease can cause increased oxidative levels) and patients with prolonged infections, such as HIV and tuberculosis patients, were omitted from the study. Total 120 patients underwent the study, among them 90 patients were diabetes patients (case group), and 30 were healthy volunteers (control group).

\section{Sources of data}

A suitable data collection protocol was designed to collect, document, and analyze the data. Informed consent section was also included in data collection form. Data collection form included demographics previous illness, history, drug usage pattern, and details of patients were collected from the patient's case notes, treatment chart, nurse's notes, laboratory reports, out patient records, by interviewing patients and/ or their caretakers, and health-care professionals, wherever necessary. All the collected data were documented in a suitably designed data collection form developed for the study.

\section{Sample collection}

- Blood has been collected from the patients subsequently got the informed consent from the patient or the attendee.

- Centrifugation of the sample was done at a speed of $3000 \mathrm{rpm}$ for $30 \mathrm{~min}$, and the greater layer is divided in labeled Eppendorf tubes and kept at $4^{\circ} \mathrm{C}$ until biochemical analysis.

- Separated the blood cells and estimated the antioxidant level in red blood cells.

Table 1: Distribution of patients according to the sex

\begin{tabular}{llll}
\hline Variables & Diabetes patients & Healthy population & ChiQ/p-value \\
\hline Sex & & & \\
Male & $42(46.67 \%)$ & $13(43.33 \%)$ & 0.10 \\
Female & $48(53.33 \%)$ & $17(56.67 \%)$ & 0.75 \\
Total & 90 & 30 & 120 \\
\hline
\end{tabular}

\section{Biochemical assays}

SOD assay

The activity of SOD determined as per kakkar et al method, briefly RBS lysate was added to sodium pyrophosphate buffer ( $\mathrm{pH}$ 7.0) containing phenazine methosulphate, Nitro blue tetrazolium. Started reaction by adding NADH. SOD inhibits nitro blue reduction. The percentage inhibition of Nitro blue tetrazolium reduction is a measure of the activity of the SOD. Stopped the reaction after 1 minute by adding glacial acetic acid and read at $560 \mathrm{~nm}$ absorbance. Normal values: 5200-5500 U/g Hb [10].

\section{CAT assay}

Catalase in hemolysate was assayed based on its ability to decompose $\mathrm{H}_{2} \mathrm{O}_{2}$ in to water. The decrease in the $\mathrm{H}_{2} \mathrm{O}_{2}$ concentration was measured at $240 \mathrm{~nm}$. Normal values: $375-425 \mathrm{U} / \mathrm{g} \mathrm{Hb}$ [10]

\section{Glutathione peroxide assay}

Estimation of GPx activity in hemolysate was based on the method of Paglia and Valentine using hydrogen peroxide, GSH, glutathione reductase and NADPH and the rate of disappearance of NADPH at $37^{\circ} \mathrm{C}$ and was recorded spectrophotometrically $(340 \mathrm{~nm})$. Normal GPx level: $17.5-19.6 \mathrm{U} / \mathrm{g} \mathrm{Hb}$

\section{Statistical analysis}

The quantified variables in the study (age, sex, superoxide dismutase, catalase and glutathione peroxidase levels) were subjected to statistical analysis. All these values were analyzed for mean, standard deviation, errors. The data were statistically analysed using SAS (Version 9.1) statistical software. Unpaired Student's ' $\mathrm{t}$ ' test was performed to compare the levels between control and study groups. $\mathrm{p}$ value is less than 0.05 was considered significant.

\section{RESULTS AND DISCUSSION}

A total number of 120 patients/volunteers were enrolled in accordance with the inclusion criteria in the study. Of which, 90 were grouped as diabetic patients named as case group, and 30 were healthy human volunteers named as controls. The ratio between the control group and case group is 1:3. In case group, among 90 patients, 42 (46.7\%) were males and $48(53.3 \%)$ were females, and in control group, of 30 healthy population, $13(43.3 \%)$ were males and $17(56.7 \%)$ were females. The test which has been used for distributing the sex ratio is Chi-square test, and the ratio was equal it's (Chi-square value $0.10, p=0.75$ ) shown in Table 1.

Age, glycated hemoglobin (HbA1c), fasting blood sugar (FBS), and post lunch blood sugar (PLBS) levels were expressed in mean and standard deviation and to compare differences above variables in both groups and unpaired t-test used, and a minimum level of significant was $<0.05$, details are shown in Table 2. The means of age in case and control groups were $53.3 \pm 8.2$ and $55.6 \pm 7.2$, respectively. It shows (t-value 1.4 , $\mathrm{Z} \mathrm{p}=0.16$ ) no differences.

The mean \pm standard deviation of HbA1c in case and control groups was $10.9 \pm 1.8$ and $4.71 \pm 0.98$, respectively. The statistically shown ( $\mathrm{t}$-value

Table 2: Compare the character of between diabetes patients and health populations

\begin{tabular}{|c|c|c|c|c|}
\hline Sl. No & Variable & Diabetes group & Healthy group & t value $p$-value \\
\hline & Age in years & & & \\
\hline \multirow[t]{2}{*}{1} & Mean \pm SD & $56.25 \pm 8.16$ & $55.5 \pm 7.19$ & 1.380 .16 \\
\hline & HbA1c (\%) & & & \\
\hline \multirow[t]{2}{*}{2} & Mean \pm SD & $10.90 \pm 1.81$ & $4.71 \pm 0.98$ & $-18.51<0.0001$ \\
\hline & Fasting blood glucose mg/dL & & & \\
\hline \multirow[t]{2}{*}{3} & Mean \pm SD & $154.58 \pm 15.98$ & $88.36 \pm 5.30$ & $-22.01<0.0001$ \\
\hline & Postprandial Blood Glucose (mg/dl) & & & \\
\hline 4 & Mean \pm SD & $242.25 \pm 26.44$ & $135.25 \pm 10.47$ & $-21.23<0.0001$ \\
\hline
\end{tabular}

HbA1c: Glycated hemoglobin, SD: Standard deviation 
$18.5, \mathrm{p}<0.0001)$ differences are highly significant. The mean \pm standard deviation of FBS in case and control groups was 154.6 \pm 16.0 and $88.4 \pm 5.3$, respectively. It shown ( $t$-value- $22.0, p<0.0001$ ) differences is highly significant.

PLBS levels in case group and control group were $245 \pm 26.44$ (mean \pm SD) and $132.25 \pm 10.47($ mean \pm SD) simultaneously. Highly significant statistical difference between case and control group as calculated by t-value-21.23, $\mathrm{p}<0.0001$.

The levels of SOD, Catalase and GPx levels were weighty low in Diabetes Mellitus (case group) compared to control group. Superoxide Dismutase levels in case group and control group were $3859.00 \pm 381.8$ (mean+SD) and $5862.7 \pm 209.45$ (mean+SD) Units per gram $\mathrm{Hb}$, respectively. The difference between case and control group as calculated by t-value (t-value 27.35, p $<0.0001$ ), it's shown statistically highly significant. Catalase levels in case group and control group were $212.7 \pm 19.08$ (mean \pm SD) and $396.47 \pm 10.83($ mean \pm SD) Units per gram $\mathrm{Hb}$, respectively. The difference between case and control group as calculated by t-value (t-value 50.07, $\mathrm{p}<0.0001$ ), it's shown statistically highly significant. Glutathione Peroxidase levels in case group and control group were $11.7 \pm 01.09$ $($ mean+SD) and 18.6 \pm 01.00 (mean $+\mathrm{SD}$ ) Units per gram $\mathrm{Hb}$, respectively. The difference between case and control group as calculated by t-value (t-value 30.26, p<0.0001), it's shown statistically highly significant. Levels observed in control group were in physiological normal range. It is indicative from this study that diabetes mellitus patients have low levels of antioxidants compared to healthy population. Low levels of antioxidants leads to oxidative stress in diabetes patients.

Good correlation observed between the levels of HBA1C and antioxidants, such as SOD, CAT, and GPx, which were estimated from the $\mathrm{RBC}$ of case group, shown in tables.

In 120 patients, $\mathrm{HbA1C}, \mathrm{SOD}, \mathrm{CAT}$, and GPx levels were estimated; the correlation was calculated between the HbA1c and antioxidants (SOD, CAT, and GP) in both groups, diabetes patients details are shown in Table 3.

In diabetes group exhibited a major negative correlation between HbA1c and antioxidants such as SOD $(r=-0.94, p<0.0001)$, CAT $(\mathrm{r}=-0.74, \mathrm{p}<0.0001)$, and GPx levels $(\mathrm{r}=-0.76, \mathrm{p}<0.0001)$

The antioxidants parameters, such as SOD, CAT, and GPx, were estimated. The degrees of activity of SOD, CAT, and GPx in diabetic cases and controls were compared and are shown in Table 4 and graphically in Figs. 1-3.

In health population group, showed no significant between $\mathrm{HbA1c}$ and antioxidants such as SOD ( $r=0.07, p=0.17)$, CAT ( $r=0.11, p=0.09)$, and GPx

Table 3: Pearson correlation coefficient between HbA1c and antioxidants (SOD, CAT, and GP) in diabetes patients and health population

\begin{tabular}{llll}
\hline Sl. No & Antioxidants & $\begin{array}{l}\text { HbA1c case group } \\
\text { pearson } \mathbf{r} \text { (p-value) }\end{array}$ & $\begin{array}{l}\text { HbA1c control group } \\
\text { pearson } \mathbf{r} \text { (p-value) }\end{array}$ \\
\hline 1 & SOD (U/g) & $-0.94(<0.0001)$ & $0.07(>0.17)$ \\
2 & CAT (U/g) & $-0.74(<0.0001)$ & $0.11(>0.09)$ \\
3 & GPx (U/g) & $-0.76(<0.0001)$ & $0.16(>0.07)$ \\
\hline
\end{tabular}

SOD: Superoxide dismutase, CAT: Catalase, GP: Glutathione peroxidase, HbA1c: Glycated hemoglobin levels $(\mathrm{r}=-0.16, \mathrm{p}=0.07)$. It's indicated that antioxidant levels will decrease in diabetes patient its inverse correlation with Glycated Hemoglobin. Low antioxidants levels were observed in these patients compared to healthy individuals [5]. Antioxidants are the necessary elements involved in the action of insulin and energetic metabolism without serious adverse drug reactions. The antioxidant therapy in diabetes and other disorders are debatable. To check whether diabetes mellitus needs antioxidant treatment, the following study was conducted [11]

Related studies determined that lower number of antioxidants may lead to oxidative injury in diabetic patients, Saritha et.al. In contrast with the earlier study, the focuses of SOD, CAT, and GPx were expressively low in Type II DM as matched to the control group. Individuals with diabetes and low levels of antioxidants lead to increased risk of microvascular and macrovascular [12] complications.

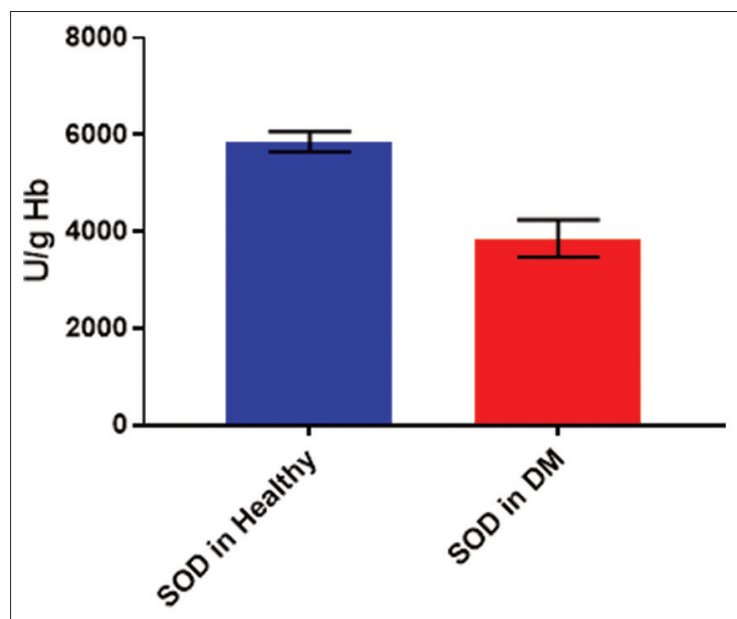

Fig. 1: Compare superoxide dismutase in both groups mean \pm standard deviation

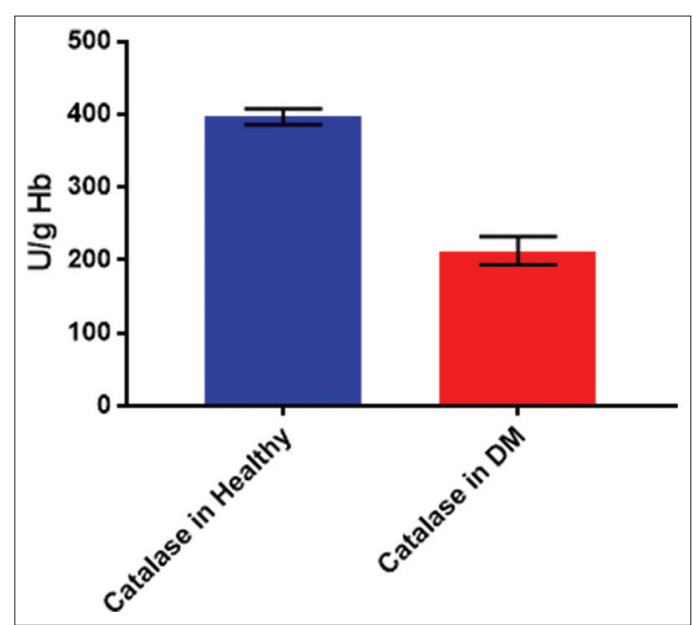

Fig. 2: Compare catalase in both groups (mean \pm standard deviation)

Table 4: Compare antioxidants levels (SOD, CAT and GPX) between diabetes patients and health populations

\begin{tabular}{lllll}
\hline Sl. No & Antioxidants & $\begin{array}{l}\text { Diabetes (Case group) } \\
\text { Mean } \pm \text { S.D }\end{array}$ & $\begin{array}{l}\text { Health population (Control group) } \\
\text { Mean } \pm \text { S.D }\end{array}$ & T-value p-value \\
\hline 1 & SOD (U/g) & $3859.00 \pm 381.8$ & $5862.7 \pm 209.45$ & $27.35<0.0001$ \\
2 & CAT (U/g) & $212.7 \pm 19.08$ & $396.47 \pm 10.83$ & $50.03<0.0001$ \\
3 & GPx (U/g) & $11.7 \pm 01.09$ & $18.605 \pm 01.00$ & $30.26<0.0001$ \\
\hline
\end{tabular}

SOD: Superoxide dismutase, CAT: Catalase, SD: Standard deviation, GP: Glutathione peroxidase 


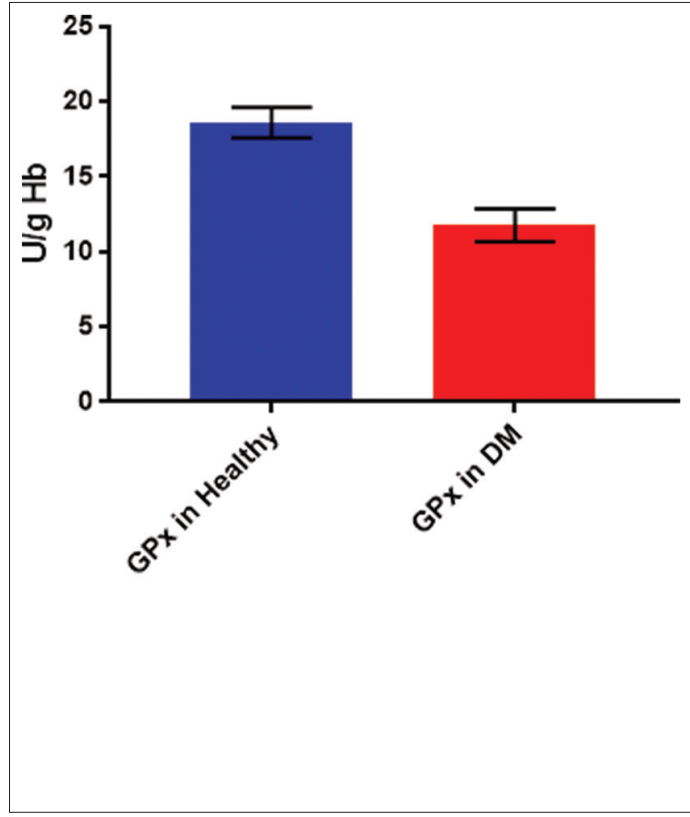

Fig. 3: Compare glutathione peroxidase in both groups (mean \pm standard deviation)

The previous experiments have published significantly low levels of antioxidants. OS is the probable cause of problems such as diabetic nephropathy in DM. The previous experiments have exposed that significantly reduced antioxidant enzyme actions may increase the support to grow the risk of complications such as macrovascular and microvascular complications [13]. Moreover, the previous study observed that there was a negative correlation between total antioxidant status and DNA damage [14].

In the current study, marked high levels of HbA1c levels in diabetic patients due to excessive glycosylation of $\mathrm{Hb}$ which is in agreement to the previous studies [15]

\section{CONCLUSION}

In summary, ROS creation overcomes the antioxidant fortifications of the cell either because of rich production of oxidants or reduction of antioxidant fortifications, several cellular constituents can be injured. The crucial cellular marks are DNA, lipids, and proteins. These macrovascular and microvascular complications are associated with cardiovascular disease, atherosclerosis, stroke, nephropathy, retinopathy, and neuropathy. The necessity of this work is to define the levels of antioxidants such as CAT, GPx, and SOD in Type II diabetic populations. Low concentration of antioxidants, such as CAT, GPx, and SOD, were observed in Type II diabetic populations. Where, the management of antioxidants supplements is wanted for the Type II diabetic populations, and it advantages the severe attention to decrease the hazard of upcoming cardiovascular complications.

\section{CONFLICT OF INTEREST STATEMENT}

There are no conflicts of interest.

\section{AUTHOR'S CONTRIBUTION}

First author (Sudharshan Reddy Nelli), his collected the data, data analysis, and interpretation and written draft copy of article.

Second author (Nilesh Kumar Sharma), his design the work guided and help to the first author to collect the data and given final approval to publish the work.

Third author (Manoj Kumar P), his help in interpretation and critical revision of the article.

Fourth author (Surya S Singh), his contributed to estimate the antioxidant levels.

\section{REFERENCES}

1. Kangralkar VA, Patil SD, Bandivadekar RM. Oxidative stress and diabetes, a review. Int J Pharm Appl 2010;1:38-45.

2. Mohan V, Sandeep S, Deepa R, Shah B, Varghese C. Epidemiology of type 2 diabetes: Indian scenario. Indian J Med Res 2007;125:217-30.

3. World Health Organization. Diabetes. Vol. 10. Geneva: World Health Organization; 2014. p. 1-3. Available from: http://www.who.int/ mediacentre/factsheets/fs312/en/.

4. Fowler MJ. Microvascular and macrovascular complications of diabetes. Clin Diab 2008;28:277-82.

5. Al-Rawi NH. Oxidative stress, antioxidant status and lipid profile in the saliva of type 2 diabetics. Diab Vasc Dis Res 2013;8:22-8.

6. Matough FA, Budin SB, Hamid ZA, Alwahaibi N, Mohamed J. The role of oxidative stress and antioxidants in diabetic complications. SQU Med J 2012;12:5-18

7. Maharajan BR, Jha JC, Vishwanath P, Adhikari D, Baxi J, Alular VM, et al. A study of oxidative stress, antioxidant status and lipid profile in diabetic patient in the western region of Nepal. Kathmandu Univ Med J 2008;6:16-22

8. Tan SM, Sharma A, de Haan JB. Oxidative stress and novel antioxidant approaches to reduce diabetic complications. Oxidative Stress Dis 2012;16:247-80. Available from: http//www.intechopen.com.

9. Merzouk S, Hichami A, Madani S, Prost J, Khan NA. Antioxidant status and levels if different vitamins determined by high performance liquid chromatography in diabetic subjects with multiple complications. Gen Physiol Biophys 2003;22:15-27.

10. Sangram V, Manasaveena V, Akula KK. A comparative evaluation of enzymatic antioxidant levels in pre and post therapy patients with oral cancer. Int J Pharm Pharm Sci 2014;6(11):52-6.

11. Shinde SN, Dhadke VN, Suryakar AN. Evaluation of oxidative stress in type 2 diabetes mellitus and follow-up along with Vitamin E supplementation. Indian J Clin Biochem 2011;26:74-7.

12. Kasznicki J, Kosmalski M, Sliwinska A, Mrowicka M, Stanczyk M. Evaluation of oxidative stress markers in pathogenesis of diabetic neuropathy. Mol Biol Rep 2012;39:8669-78.

13. Pasupathi P, Chandrasekar V, Senthilkumar U. Evaluation of oxidative stress, antioxidant and thyroid harmone status in patients with diabetes mellitus. J Medicine 2009;10:60-6.

14. Kolgiri VV, Patil W, Nagar V. Correlation of total antioxidant status (Tas) with dna damage in HIV/AIDS patients. Int J Pharm Pharm Sci 2016;8(6):240-4.

15. Niedowicz DM, Daleke DL. The role of oxidative stress in diabetic complications. Cell Biochem Biophys 2005;43:60-6.

16. Yang H1, Roberts LJ, Shi MJ, Zhou LC, Ballard BR, Richardson A, Guo ZM. Retardation of atherosclerosis by overexpression of catalase or both $\mathrm{Cu} / \mathrm{Zn}$-superoxide dismutase and catalase in mice lacking apolipoprotein. E Circ Res 2004;95(11):1075-81

17. Omolola R, Ayepola, Nicole L, Brooks, Oluwafemi O, Oguntibeju. Oxidative stress and diabetic complications: The role of antioxidant vitamins and flavonoids. Chapter 2:25-58. Available from: http:// dx.doi.org/10.5772/57282. 